\title{
A systematical veto by all monitor signals in a gravitational-wave detector
}

\author{
Koji Ishidoshiro ${ }^{1}$, Masaki Ando ${ }^{1}$, Kimio Tsubono ${ }^{1}$, Nobuyuki Kanda ${ }^{2}$ \\ and Hirotaka Takahashi ${ }^{3}$ (and the TAMA Collaboration) \\ ${ }^{1}$ Department of Physics, The University of Tokyo, 7-3-1 Hongo, Bunkyo-ku Tokyo 113-0033, \\ Japan \\ 2 Department of Physics, Graduate School of Science, Osaka City University, Osaka 558-8585, \\ Japan \\ ${ }^{3}$ Max-Planck-Institut für Gravitationsphysik, Albert-Einstein-Institut, Am Mühlenberg 1, \\ D-14476 Golm bei Potsdam, Germany \\ E-mail: koji_i@granite.phys.s.u-tokyo.ac.jp
}

Received 30 March 2007, in final form 6 July 2007

Published 19 September 2007

Online at stacks.iop.org/CQG/24/S405

\begin{abstract}
We present the current state of veto studies in TAMA300 by monitor signals of the interferometer and its environment. In GW data analysis, fake GW events may bury real GW events or worse upper limits on the event rate. Thus, fakeevent rejection is an important issue. In general, we can reject these fake events by the monitor signals, since these fake events are induced due to detector instabilities. However, using all monitor signals for the fake-event rejection would increase the accidental rejection probability and dead time without improving veto efficiency, since all monitor signals do not have correlations to the detector instabilities. Here, we analyze coincidences between the main and selected monitor signals with the optimal parameters for the fake-event rejection. Then, coincident events are rejected as the fake events. For the signal selection and parameter optimization, we systematically investigate the correlations with the detector instabilities. As a result, we achieved 30-99\% veto efficiency using ten selected monitor signals with the $3.2 \%$ accidental rejection probability and $0.2 \%$ dead time.
\end{abstract}

PACS numbers: $04.80 . \mathrm{Nn}$, 07.05.Kf, 95.55.Ym

(Some figures in this article are in colour only in the electronic version)

\section{Introduction}

Several interferometric gravitational-wave (GW) detectors have started searching for GW signals [1-4]. One of the most promising signals for these detectors are burst GWs from transient astrophysical phenomena, such as supernova explosions, $\gamma$-ray bursts and mergers 
of binary systems. Unfortunately, we cannot use a matched filtering method for the detection of these burst GWs, since we only have a little knowledge on the burst waveforms. Instead, several burst filters have been proposed to extract non-Gaussian or non-stationary components as burst events in the stationary-Gaussian-noise background: an excess power filter [5], a wavelet-based power filter [6], a slope filter [7] and a cluster filter in the time-frequency plane [8].

These burst filters may extract fake events from non-Gaussian or non-stationary noise. These fake events may bury real GW events. Thus, it is a critical problem for burst data analysis to reject the fake events among detected burst events $[9,10]$. The most simple scheme to reject the fake events is coincidence analysis with multiple GW detectors [11]. If we detect burst events with multiple GW detectors exceeding an acceptable time difference, these burst events are rejected as fake events. On the other hand, we can reject the fake events with a single detector by waveform behaviors [12] or interferometer and environmental monitor signals $[13,14]$. Even in the coincidence analysis, the fake-event rejection with a single detector is important, since it would reduce an accidental coincidence probability.

In this paper, we present the current state of veto studies by the monitor signals in TAMA300. TAMA300 is a GW detector in Japan (see section 3). The monitor signals are recorded together with a main signal (the feedback signal of the differential motion of the arm cavities), which might contain GW signals, to watch the detector instabilities and environmental fluctuations. When the main and monitor signals have burst events at the same time, we can reject them as fake events induced by the detector instabilities. In our previous studies [9, 14], limited monitor signals were used for the fake-event rejection. These analyses have the possibility of missing unknown correlations between the monitor signals and the detector instabilities. On the other hand, using all monitor signals would increase an accidental rejection probability or dead time without any improvement of veto efficiency. Here, we systematically select correlated monitor signals and optimize analysis parameters. Then, we use selected monitor signals with optimal parameters for the fake-event rejection.

\section{Method}

\subsection{Overview}

In this section, we overview our veto method. Figure 1 shows a schematic outline. The left box in figure 1 shows the scheme of the fake-event rejection by selected monitor signals with optimal parameters. The right box in figure 1 shows the data flow of the systematic investigation of all monitor signals for signal selection and parameter optimization. First, we apply a data conditioning and a burst filter to the main signal. The data-conditioning filter removes line noises and normalizes the signal power. The burst filter extracts nonGaussian or non-stationary components as burst events. Details of each filter are described in sections 2.2 and 2.3. Second, the same process is repeated on selected monitor signals with optimal parameters. Finally, we analyze coincidences between the main and selected monitor signals for the fake-event rejection. If burst events of the main signal are coincident with them of the selected monitor signals, they are rejected as the fake events. If they are not, they are recorded as GW candidates. Detail of these steps, often called an 'event-by-event veto', is explained in section 2.4. In section 2.5, we systematically select the monitor signals used for the fake-event rejection and optimize the analysis parameters to obtain the highest veto efficiency, using $10 \%$ of the total data. We call the data 'playground data', only used for the signal selection and parameter optimization. Here, the veto efficiency is the probability that the burst events of the main signal are rejected. 


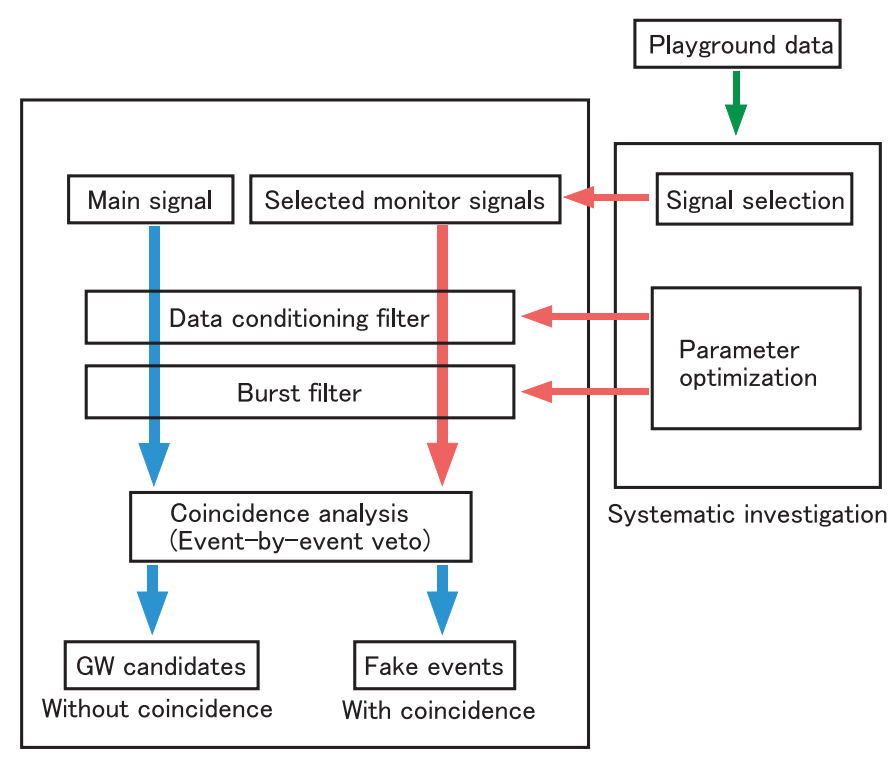

Fake rejection

Figure 1. Schematic outline of our systematic veto. The left schematic shows the fake-event rejection by the monitor signals. The right schematic shows the systematically investigation for the signal selection and parameter optimization.

\subsection{Data-conditioning filter}

In the first step of the analysis, a data-conditioning filter is required, since raw data are not ideal stationary-Gaussian noise; the noise spectrum is not white, the noises level changes in time, and many line peaks (harmonics of $50 \mathrm{~Hz} \mathrm{AC}$ line, violin-mode peaks of the suspension wire of the mirror and a calibration peak of $625 \mathrm{~Hz}$ ) are included in the power spectrum. The data-conditioning filter is composed of the following steps. (i) The data are split into segments of length $\Delta T=72.0896 \mathrm{~s}$ with an overlap of $(3.2678 \mathrm{~s})$. Below, we consider one $\Delta T=72.0896 \mathrm{~s}$ data. (ii) The complex Fourier components $\left(\tilde{V}^{j}\right)$ are calculated from timeseries signal $\left(V^{i}\right)$ using a fast Fourier transform (FFT). The indices $i$ and $j$ show the time and the frequency. (The indices $i, j=1,2, \ldots, 2^{16} \times 22$ for HDAQ signals, $i, j=1,2, \ldots, 2^{10} \times 22$ for MDAQ signals, HDAQ and MDAQ are explained in section 3.1.) (iii) Line peaks are removed by $\tilde{V}^{j_{m}}=0$, where $j_{m}$ is the corresponding line frequency. (iv) The lower frequency components below the cut-off frequency $f_{l}$ and the higher frequency components above the cut-off frequency $f_{h}$ are removed by

$$
\tilde{V}^{j}=0 \quad(j \leqslant l \text { or } j \geqslant h) .
$$

The indices $l$ and $h$ correspond to the cut-off frequencies. (v) The complex Fourier components are normalized (whitened) by the mean noise spectrum before $10 \mathrm{~min}$ by

$$
\tilde{V}^{\prime j}=C \frac{\tilde{V}^{j}}{\sqrt{\left\langle\left|\tilde{V}^{j}\right|^{2}\right\rangle_{10 \min }}} .
$$

Here, $C$ is the normalization constant. (vi) A band-limited and normalized time-series signal $\left(x_{l h}^{I}\right)$ is calculated from the normalized components $\left(\tilde{V}^{\prime j}\right)$ with an inverse FFT. Data (3.2678 s) of each edge are thrown away, since there is an error caused by a finite Fourier transform. The signal $\left(x_{l h}^{I}\right)$ is composed of 65.536 s. $I$ is the new time index $\left(I=1,2, \ldots, 2^{16} \times 20\right.$ 
for HDAQ signals, $I=1,2, \ldots, 2^{10} \times 20$ for MDAQ signals) and $C$ is fixed so that $\left\langle\left(x_{l h}^{I}\right)^{2}\right\rangle=1$.

For the main signal, we use the cut-off frequencies $800 \mathrm{~Hz}$ and $2000 \mathrm{~Hz}$, since the 800-2000 Hz band corresponds to around the floor level in TAMA300. For a monitor signal, which we call the $k$ th monitor signal, we use the optimal cut-off frequencies $\left(l_{k}, h_{k}\right)$, since each signal has different correlations to the instabilities. The optimized process is explained in section 2.5.

\subsection{Burst filter}

We use a excess-power statistic as the detection statistic in our burst filter since the power has a high sensitivity to non-Gaussian or non-stationary components. The excess-power statistic is an averaged signal power in a given time-frequency window of time-frequency space [5]. Here, we calculate the averaged power $\left(\Lambda_{n}\right)$ from the band-limited signal $\left(x_{l h}^{I}\right)$, obtained by the data-conditioning filter described in section 2.2,

$$
\Lambda_{n}=\frac{\sum_{I=N n / 2+1}^{I=N n / 2+N}\left(x_{l h}^{I}\right)^{2}}{N} .
$$

Here, $N$ is the time window to be taken by the averaged power. $n$ is the time index of the time window. $\Lambda_{n}$ have $50 \%$ overlap of the previous $\Lambda_{n-1}$ for non-dismissal of burst events. Note, since the signal is normalized $\left(\left\langle\left(x_{I h}^{I}\right)^{2}\right\rangle=1\right), \Lambda_{n}$ represents the signal-to-noise ratio (SNR). If $\Lambda_{n}$ is larger than a given threshold $\left(\Lambda_{\text {thresh }}\right)$, we record $\Lambda_{n}$ as a burst event. When burst events are overlapped or continued, we treat them as one event. Then, the maximum $\Lambda_{n}$ is taken as the signal power of overlapped or continued burst events. The time window $\left(N^{0}\right)$ for the main signal is selected to be $12.8 \mathrm{~ms}\left(N^{0}=2^{8}\right.$ points with $20 \mathrm{kHz}$ sampling), which is close to the time scale of burst GWs from supernova explosions. For the $k$ th monitor signals, we select the optimal time window $\left(N^{k}\right)$, since each signal has different correlations to the instabilities. For example, the laser power may have spikelike correlations or the seismic motion may have long correlations. In addition, threshold for the $k$ th monitor signal $\left(\Lambda_{\text {thresh }}^{k}\right)$ is set so that the accidental rejection probability is $0.1 \%$. Optimization for the time window $\left(N^{k}\right)$ and the threshold $\left(\Lambda_{\text {thresh }}^{k}\right)$ is described in section 2.5.

\subsection{Coincidence analysis}

Here, we describe the method of coincidence analysis between the main and the $k$ th monitor signals. We evaluate whether each burst event of the main and the $k$ th monitor signals have the same time segment or not (see figure 2). In this analysis, the time-series data are split into segments labeled $p$. Each segment has duration of $6.4 \mathrm{~ms}^{4}$. When the burst event of the main signal and the burst event of the $k$ th monitor signal have at least one identical segment, we regard that the burst events have coincidence. The burst events with coincidences are rejected as the fake events induced by the detector instabilities. If there are no coincidences, we record the burst events as GW candidates. The cumulative time of overlapping the segments, called dead time, is not used for setting upper limits. The above process is repeated for other monitor signals.

Figure 2 is an example of the coincidence analysis. In this case, the main signal has the burst event $\mathrm{A}$ at $p=5,6,7,8$ and the $k$ th monitor signal has the burst event $\mathrm{B}$ at $p=1,2$ and burst event $\mathrm{C}$ at $p=7,8,9,10$. Then the burst events $\mathrm{A}$ and $\mathrm{C}$ are coincident, since they share the segment $p=7,8$. Thus, the burst event $\mathrm{A}$ is rejected and the segments $p=5,6,7,8$

\footnotetext{
4 The duration $6.4 \mathrm{~ms}$ is our time resolution obtained by the minimum time window.
} 


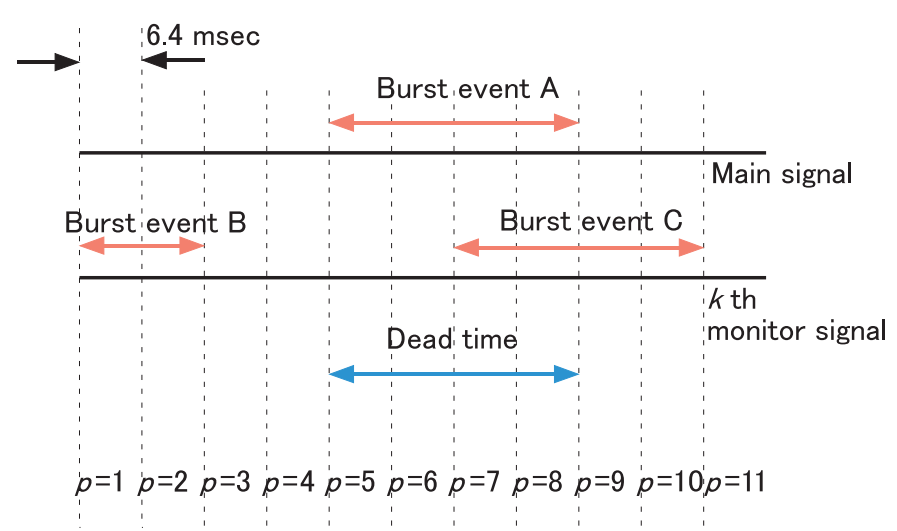

Figure 2. Example of coincidence analysis. In this case, the burst events $\mathrm{A}$ and $\mathrm{C}$ are coincident. Thus, the burst event $\mathrm{A}$ is rejected as a fake event. The segments $p=5,6,7,8$ are dead time, which are not used for setting upper limits.

are the dead time. In addition, to calculate the accidental rejection probability we analyze the coincidences between the main and the 1 min time-shifted $k$ th monitor signals.

\subsection{Systematic investigation}

We systematically select the monitor signals and optimize the analysis parameters used for the fake-event rejection by analyzing the playground data. The playground data are used only for this systematic investigation.

2.5.1. Parameter optimization. First, we show the parameter optimization of the $k$ th monitor signal. The optimized parameters are low and high cut-off frequencies $\left(l_{k}, h_{k}\right)$, time window $\left(N^{k}\right)$ and signal power threshold $\left(\Lambda_{\text {thresh }}^{k}\right)$. The optimization of these parameters is in the Neyman-Person sense [15]; the optimal parameters are selected to give the highest veto efficiency with a fixed accidental rejection probability. The accidental rejection probability is the probability that the burst events of the main signals have accidental coincidences. This probability is calculated by 1 min time-shifted analysis ${ }^{5}$. Below we show the details of parameter optimization: (i) We apply the data conditioning and burst filter to the main signal with a fixed power threshold $\left(\Lambda_{\text {thresh }}^{0}=3\right)$. This threshold corresponds to one fake event per used data (about $200 \mathrm{~h}$ (see section 3.1)) in the assumption of stationary-Gaussiannoise background. (ii) From the three-dimensional parameter space (low and high cut-off frequencies $\left(l_{k}, h_{k}\right)$ and time window $\left.\left(N^{k}\right)\right)$, we choose parameter candidates. Note, one half of this space is not valid, since $l_{k} \leqslant h_{k}$ is requested. (iii) We apply the data conditioning and the burst filter to the $k$ th monitor signal with the parameter candidates. (iv) The veto efficiency and the accidental rejection probability are calculated as a function of the threshold for the $k$ th monitor signal $\left(\Lambda_{\text {thresh }}^{k}\right)$. Here, the accidental rejection probability is calculated by the time-shift analysis. (v) We fix the threshold, corresponding to the $0.1 \%$ accidental rejection probability. From this threshold, we obtain the veto efficiency. (vi) Processes (ii)-(v) are repeated 100 times with different parameter candidates. (vii) Parameter candidates that have the highest veto efficiency are selected as the optimal parameters. (ix) Processes (ii)-(vii) are repeated for all monitor signals.

5 We did not find significant differences in these probabilities by 30 times (1-30 min) time-shifted analysis. Thus, we use the $1 \mathrm{~min}$ time shift on the monitor signals as the time-shifted analysis. 
2.5.2. Signal selection. Next, we describe the signal selection. We must select the monitor signals used for the fake-event rejection, because using the monitor signals makes the total accidental rejection probability and the dead time increase without any improvement of the veto efficiency. In addition, we would like to use the monitor signal with the high veto efficiency more effectively. Thus the monitor signals are divided into three types by the veto efficiency. (i) The monitor signals with the veto efficiency smaller than $0.5 \%$ are not used for the fake-event rejection. The ratio $0.5 \%$ is selected so that the accidental probability and veto efficiency are significantly different, since the statistical error of them is about $0.1 \%$. (ii) The monitor signals with $0.5-2.0 \%$ veto efficiency are used for the fake-event rejection. (iii) The monitor signals with the veto efficiency larger than $2.0 \%$ are used for the fake-event rejection with re-optimization of the analysis parameters for a more intensive veto. In type (iii), the parameter optimization is repeated to have lower thresholds, corresponding to the $0.5 \%$ accidental rejection probability.

\section{Application to TAMA300}

\subsection{TAMA300 data}

We apply the method of the systematic veto to data from TAMA300. TAMA300 is a laser interferometric GW detector at the Mitaka campus of the National Astronomical Observatory of Japan (NAOJ). The data used in this work were about $200 \mathrm{~h}$ of the data collected during the ninth data-taking run (DT9) from November 2003 to January 2004 [4]. For the systematic veto, 64 monitor signals were used. Three monitor signals were recorded by high-speed-dataacquisition system (HDAQ) with $20 \mathrm{kHz}$ sampling, in 16 bit resolution. Another 61 monitor signals were recorded by the middle-speed-data-acquisition system (MDAQ) with $312.5 \mathrm{~Hz}$ sampling, in 16 bit resolution.

\subsection{Safety of the veto}

For the fake-event rejection by the monitor signals, we should confirm the safety of the veto. If the detector instabilities are caused by huge GWs, we may reject real GW events. During DT8 (February-April 2003) and March 2006, we swung the interferometer mirrors with sineGaussian waveforms and typical burst waveforms from supernova explosions, obtained by numerical simulations $[16,17]$, to investigate the response of the detector (called 'hardware injection tests'). If huge GWs cause the detector instabilities, the veto efficiency must be different from the accidental rejection probability during these tests. As with the results, we did not find any differences exceeding $3 \sigma$, except for the seismic motion in DT8. We considered that this correlation was induced by human action. Actually, by remote test in March 2006, we did not find differences exceeding $3 \sigma$. Thus, we concluded that our veto was safe.

\section{Results}

As the results of the systematic investigation of all monitor signals in TAMA300, ten monitor signals were selected: laser intensity, common motion in the arm cavities and dark-port power in HDAQ, laser intensity, error and feedback signals of the differential motion from the beamsplitter to the front mirrors, bright-port power, transmitted power in the end mirrors, vertical seismic motion and magnetic field in MDAQ. The parameters of the laser intensity signals in HDAQ and MDAQ were re-optimized to have the lower threshold. For the fake-event rejection, we analyzed the coincidences between the main and ten selected monitor signals with the optimal parameters. In figure 3 , the event rates are plotted as a function of the power 


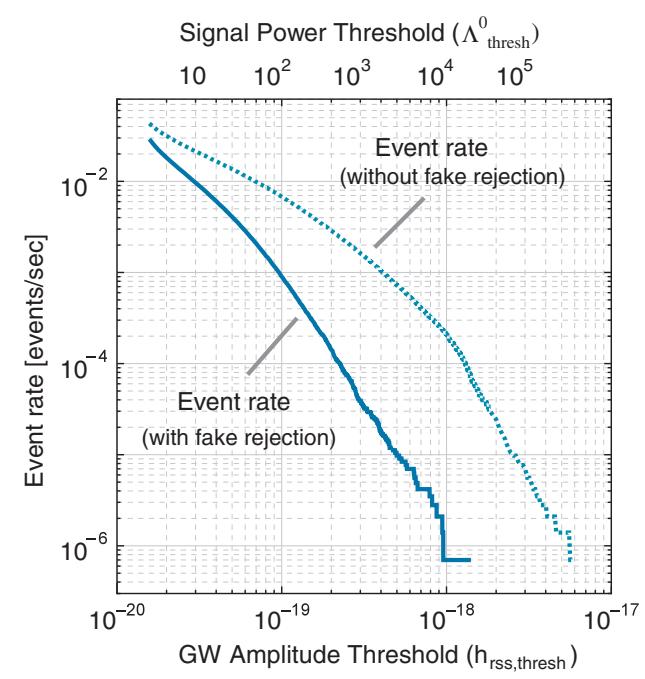

Figure 3. Event rate with and without the fake-event rejection. The horizontal axis is the power threshold $\left(\Lambda_{\text {thresh }}^{0}\right)$ or the GW amplitude $\left(h_{\mathrm{rss}}\right)$. The vertical axis is the event rate.

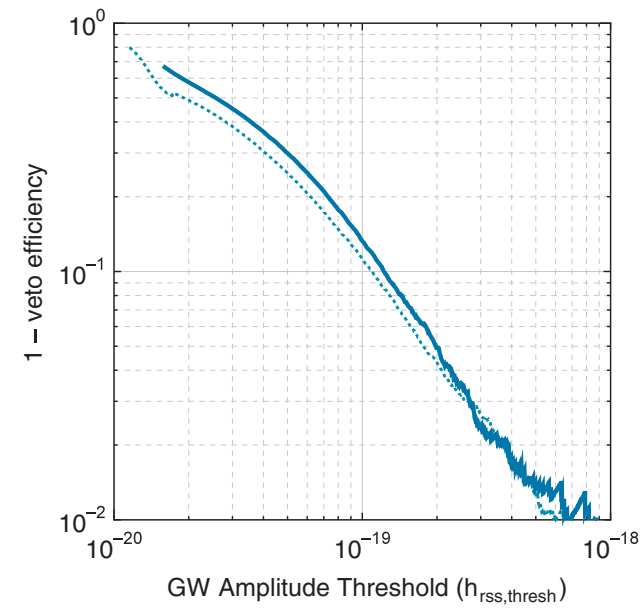

Figure 4. The veto efficiency described as a function of the GW amplitude threshold $\left(h_{\mathrm{rss}, \text { thresh }}\right)$. The solid line is our result. The dotted line represents our previous work [9].

threshold $\left(\Lambda_{\text {thresh }}^{0}\right)$ with and without the fake-event rejection. The event rate was reduced by $7 / 10-1 / 100$ with a $3.2 \%$ accidental rejection probability or $0.2 \%$ data dead time.

\section{Discussion}

We now discuss the benefits of our veto results. For the discussion, we describe the total veto efficiency as a function of the GW amplitude threshold. First, the signal power threshold $\left(\Lambda_{\text {thresh }}^{0}\right)$ is calibrated to root-sum-square amplitude $\left(h_{\mathrm{rss}}\right)$ of GWs based on the results of signal injection tests (called software-injection tests). Details of the software-injection tests are described in the appendix. Using this, the event rates in the main signal are plotted as the function of the GW amplitude threshold ( $h_{\text {rss, thresh }}$ ) in figure 3 . The veto efficiency is plotted as a function of the GW amplitude threshold in figure 4. This figure shows that our fake-event 


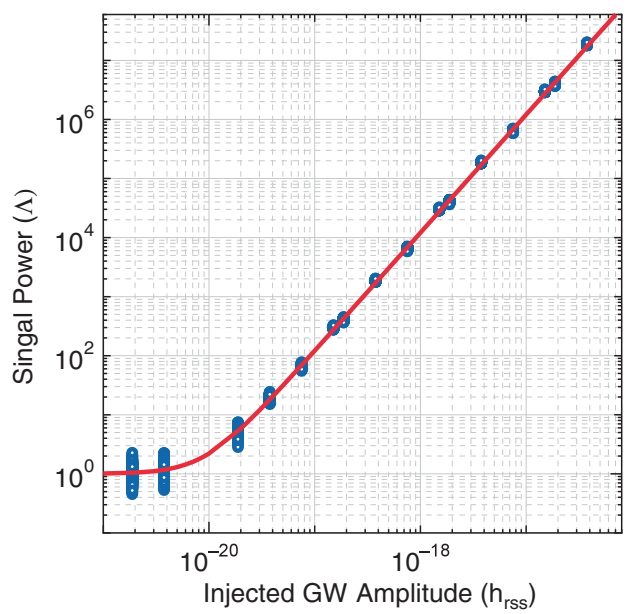

Figure A1. Result of the software-injection tests. The horizontal axis is the GW amplitude $\left(h_{\mathrm{rss}}\right)$ of the injected signal and the vertical axis is the signal power $(\Lambda)$. The solid line is the fitting result.

rejection becomes effective for loud events ${ }^{6}$. Here, we regard all detected burst events as fake events. For huge GW events $\left(h_{\mathrm{rss}} \sim 10^{-18}\right)$, the false alarm rate is decreased by about $1 / 100$. When we consider a small false alarm rate $\left(\sim 10^{-6}\right.$ [event s $\left.\left.{ }^{-1}\right]\right)$, we can reduce the threshold by about $1 / 4$.

Our method is also helpful for the detector characterization, even though the main purpose is the fake-event rejection. For example, we found an interesting correlation between the main and the magnetic-field signal, which was not recognized before these studies. Besides, information of the dead time is useful for another GW analysis.

\section{Summary}

We systematically selected ten highly correlated monitor signals and optimized the analysis parameters for the fake-event rejection in TAMA300. We achieved 30-99\% veto efficiency with the $3.2 \%$ accidental rejection probability or $0.2 \%$ dead time. Our analysis could be interpreted as detector characterization: a search for any unexpected correlation and dead time of the detector. This analysis will be applied to the next data-taking run of TAMA300.

\section{Acknowledgments}

KI would like to thank the internship program of the Initiative Program of Education for Graduate School of Science, The University of Tokyo.

\section{Appendix. Software-injection tests}

In this appendix, we discuss the software-injection tests and how to calibrate signal power $(\Lambda)$ to the root-sum-square amplitude. In our analysis, we superimposed the sine-Gaussian signals to the main signal after proper calibration. The sine-Gaussian signals are given by

$$
h\left(t+t_{0}\right)=h_{0} \sin \left(2 \pi f_{0} t\right) \exp \left(-t^{2} / \tau^{2}\right)
$$

6 It is difficult to compare between this result and our previous work [9]. For plain comparison, the veto efficiency was slightly worse. This was caused by the differences in the analyzed frequency ranges. 
where $f_{0}$ is called the central frequency and $h_{0}$ is the amplitude. The root-sum-square (rss) amplitude is defined by

$$
h_{\mathrm{rss}}=\sqrt{\int|h|^{2} \mathrm{~d} t}=\sqrt{\frac{Q}{4 \sqrt{\pi} f_{0}}} h_{0} .
$$

Here, $Q=\sqrt{2} \pi \tau f_{0}=8$.9. Sine-Gaussian signals are characterized by the central frequency $f_{0}=850,1304 \mathrm{~Hz}$ and the rss amplitude $\left(h_{\mathrm{rss}}\right)$. These data were analyzed by the same burst filter. The results of the signal injection tests are shown in figure A1. The horizontal axis shows the GW amplitude $\left(h_{\mathrm{rss}}\right)$ of the injected signal, and the vertical axis shows the signal power $(\Lambda)$. This result was fitted by

$$
\Lambda=1+\left(C \times h_{\mathrm{rss}}\right)^{2}
$$

where $C=1.6 \times 10^{20}$. We can calibrate from $\Lambda$ to $h_{\mathrm{rss}}$ by (A.3). The stationary noise corresponded to $h_{\mathrm{rss}, \text { noise }}=7.0 \times 10^{-21}\left[\mathrm{~Hz}^{-1 / 2}\right]$.

\section{References}

[1] Waldman S J 2006 Class. Quantum Grav. 23 S653

[2] Acernese F et al 2006 Class. Quantum Grav. 23 S635

[3] Hild S 2006 Class. Quantum Grav. 23 S643

[4] Ando M (the TAMA Collaboration) 2001 Class. Quantum Grav. 22 S881

[5] Anderson W G et al 2001 Phys. Rev. D 68042003

[6] Klimenko S and Mistelmakher G 2001 Class. Quantum Grav. 21 S1819

[7] Pradier T et al 2002 Phys. Rev. D 63042002

[8] Sylvestre J 2002 Phys. Rev. D 66102004

[9] Ando M et al 2005 Phys. Rev. D 71082002

[10] Abbott B et al 2006 Class. Quantum Grav. 23 S29

[11] Abbott B et al 2005 Phys. Rev. D 72122004

[12] Ando M et al 2003 Class. Quantum Grav. 20 S697

[13] Credico A D 2005 Class. Quantum Grav. 22 S1051

[14] Ishidoshiro K et al 2006 J. Phys. Conf. Ser. 3223

[15] Neyman J and Pearson K 1763 Philos. Trans. R. Soc. 53370

[16] Zwerger T and Müller E 1997 Astron. Astrophys. 320209

[17] Müller E et al 2004 Astrophys. J. 603221 